\title{
DESIGN OF M-CHANNEL PSEUDO NEAR PERFECT RECONSTRUCTION QMF BANK FOR IMAGE COMPRESSION
}

\author{
Anamika Jain ${ }^{1}$ and Aditya P. Goel ${ }^{2}$ \\ ${ }^{1}$ Department of Electronics \& Communication Engineering, M.A.N.I.T., Bhopal, India \\ anamikajain.bplegmail.com \\ ${ }^{2}$ Department of Electronics \& Communication Engineering, M.A.N.I.T., Bhopal, India \\ adityagoel2@rediffmail.com
}

\begin{abstract}
This paper proposes particle swarm optimization method to design M channel near perfect reconstruction pseudo QMF banks used in transforming stage of image coder. The filter bank is designed to have highest entropy based coder. To achieve high energy compaction and least distortion, design problem is formulated as a combination of the coding gain, low dc leakage conditions and stopband attenuation. For distortion free signal representation perfect reconstruction and good visual quality measures are imposed as constraints. The design problem is solved using (particle swarm optimization) PSO technique for minimizing filter tap weights. The technique find out solution by searching feasible solutions that achieve the best solution for the objectives criteria mentioned above. The performance of this optimization technique in filter bank design for image compression is evaluated in terms of both objective quality via coding gain, PSNR measures and subjective visual quality measure using both JPEG baseline image coder and an Embedded Zerotree Wavelet (EZW) coder. For comparison same test images for approximately same conditions and characteristics are used to measure compression ratio and peak signal to noise ratio (PSNR) for lower bit rates.
\end{abstract}

\section{Keywords}

Bits per pixel (bpp), Embedded Zerotree Wavelet (EZW), evolutionary Algorithm, Huffman Encoder, near perfect reconstruction, Peak signal to noise ratio (PSNR), Pseudo QMF bank, Particle Swarm optimization(PSO), reconstruction error $(R E)$.

\section{INTRODUCTION}

EZW is computationally very fast and among the best image compression algorithm known today [1]-[2]. Multirate filter banks are an important part of the image compression since a long time. As the time progresses research efforts are made to get more and more compression ratio with perfect reconstruction or near perfect reconstruction. In image compression block transform and subband coding are two most popular techniques [3]-[5]. First technique uses $\mathrm{M}$ channel $\mathrm{M}$ taps linear phase paraunitary filter bank (DCT), while the other one use wavelets [6]. In Standard JPEG (based on DCT) input image is transformed into decorrelated coefficients which are then processed by a quantizer, bit allocator and entropy coder to have compression with desired quality output image. M channel PRFB's enjoyed success due to their low complexity with reasonable performance. At low bit rates < (1 bpp) JPEG suffers from the blocking artifacts which can be reduced if the no. of taps in the filter banks made higher than the no. of channels. This

DOI : 10.5121/sipij.2012.3408 
Signal \& Image Processing : An International Journal (SIPIJ) Vol.3, No.4, August 2012

development results in lapped orthogonal transform [7]. As the length of the subband filters changes we get changes in the performance of the filter bank. When such filter banks replace dyadic wavelet transform in EZW coder the coding performance of the system changes drastically [8] i.e. coding gain and PSNR improves at lower bit rates as well.

This paper proposes design of $\mathrm{M}$ channel pseudo QMF filter banks in EZW coder as a replacement of DWT transform in combination with Huffman encoder for image compression. As the transform reduce the size of resultant data in encoder and combine and reconstruct the original data in decoder, well-optimized filter banks play an integral role in the coder's performance. A number of examples are given to show the superiority of the designed filter bank in terms of Compression Ratio (CR) and Peak- Signal to Noise (PSNR) for different threshold values [9].

In this paper, an approach based on particle swarm optimization technique (PSO) is used to find the optimizing coefficients of prototype filter to have high stopband attenuation, higher coding gain and lower overall amplitude distortion characteristic [10].

The outline of the paper is as follows: in Section 2, a relevant brief analysis of the pseudo QMF bank is given. Section 3 describes the formulation of the design of high-performance transforms to implement as block transform in the new ezw coding scheme. A brief overview of PSO technique is explained in Section 4. Section 5, presents coding examples to illustrate the validity of the proposed technique. A performance comparison between the new coder and existing standard JPEG (dct based) and ezw (wavelet based) coder is also included. Finally, the conclusions are drawn in Section 5.

\section{PSEUDO QMF BANK DESCRIPTION}

A typical M-channel filter bank is shown in Figure 1. The input signal $x(n)$ is decomposed into $M$ subband having equal band width, using the low-pass, bandpass and high-pass analysis filters $\mathrm{H}_{0}(\mathrm{z}), \mathrm{H}_{1}(\mathrm{z}), \ldots \mathrm{H}_{\mathrm{M}-1}(\mathrm{z})$ respectively. These Subband signals are down sampled by a factor of $\mathrm{M}$ and processed to transmission. At the output end, the subband signals are combined after interpolation by a factor of $\mathrm{M}$ and filtered via synthesis filters, $\mathrm{F}_{0}(\mathrm{z}), \mathrm{F}_{1}(\mathrm{z}), \ldots \mathrm{F}_{\mathrm{M}-1}(\mathrm{z})$, to have perfectly/near perfectly reconstructed output signal $x^{\Upsilon}(n)[11]$.

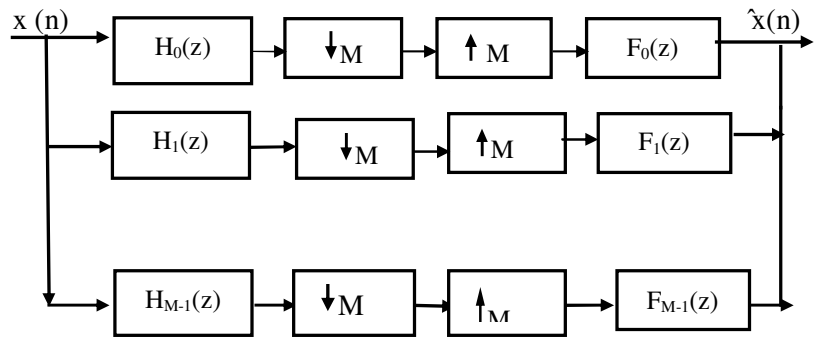

Figure $1 \mathrm{M}$-channel maximally-decimated filter bank

The reconstructed output of the filter bank is

$$
\hat{X}(z)=\frac{1}{M} T_{0}(z) X(z)+\frac{1}{M} \sum_{k=0}^{M-1} T_{r}(z) X\left(W_{M}^{r}\right)
$$

Where $\mathrm{T}_{0}(\mathrm{z})$ is a distortion transfer function and $\mathrm{T}_{\mathrm{r}}(\mathrm{z})$ is an aliasing distortion transfer function The perfect reconstruction property is satisfied when the output signal is a delayed version of the input signal i.e. $y[n]=x[n-n 0]$ for some integer $n 0$. 
The impulse response of analysis and synthesis filter derived from prototype filter $h(n)$ with the

aid of cosine modulation can be expressed as follows

$$
\begin{aligned}
h_{k}(n) & =2 h(n) \cos \left((2 k+1) \frac{\pi}{2 M}\left(n-\frac{N-1}{2}\right)+(-1)^{k} \frac{\pi}{4}\right) \\
f_{k}(n) & =2 h(n) \cos \left((2 k+1) \frac{\pi}{2 M}\left(n-\frac{N-1}{2}\right)-(-1)^{k} \frac{\pi}{4}\right)
\end{aligned}
$$

for $\mathrm{k}=0,1, \ldots(\mathrm{M}-1)$ and $\mathrm{n}=0,1, \ldots, \mathrm{N}-1$, where $\mathrm{M}$ is no. of bands and $\mathrm{N}$ is the order of the prototype filter $\mathrm{N}=2 \mathrm{mM}$ to satisfy linear phase property.

\section{PROBLEM FORMULATION}

\subsection{Filter Bank/Transform Design}

In this paper, considered M-band Pseudo QMF banks are real coefficient, maximally decimated, FIR, uniform-band filter bank with linear phase and nearly perfect reconstruction property.

The cost function used in this paper are coding gain stopband attenuation , and low dc leakage subject to perfect reconstruction violation measure all of which are well-known properties in yielding the best reconstructed image quality [12] :

$$
C_{\text {overall }}=k_{1} C_{\text {codinggain }}+k_{2} C_{\text {analysisstop }}+k_{3} C_{\text {synthesisstop }}+k_{4} C_{D C}
$$

Subject to the constraint:

$$
P R V<\beta
$$

\subsubsection{PRV Measure}

The PR property is highly desirable for a lossless signal representation; however, in image compression this condition can be relaxed to have high stopband attenuation and high coding gain.

In M-band pseudo QMF bank, perfect reconstruction conditions on the impulse response of the prototype filter $\mathrm{h}[\mathrm{n}]$ for $\mathrm{n}=0$..... $\mathrm{N}$ are

$$
\begin{aligned}
2 \sum_{r=0}^{N-2 M k} h(r) h(2 M k+r) & =\delta(k), 1 \leq m \leq \frac{M}{2} \\
0 & \leq k \leq E\left\lfloor\frac{N}{2 M}\right\rfloor
\end{aligned}
$$

Where $\delta(k)$ is the Kronecker symbol, i.e. $\delta(0)=1$ and $\delta(k)=0$ for $\mathrm{k} \neq 0$ and $\lfloor E[n]\rfloor$ is the integer part of $n[13]$.

Perfect reconstruction violation measure can be represented as PRV [14]:

$$
P R V=\sum_{k=0}^{\left.k=E \mid \frac{N}{2 M}\right\rfloor}\left(\Theta(k)-2 \sum_{r=0}^{N-2 M k} h(r) h(2 M k+r)\right)
$$




\subsubsection{Stopband Attenuation}

Stopband Attenuation of the filters in a high quality cosine modulated filter banks should be high as it measures the sum of all of the filters' energy outside the designated passband. The evaluation of the stopband energy for prototype filter on $\Omega$ leads to the following expression

$$
E_{s}=\frac{1}{\pi-\omega_{s}} \int_{\omega_{s}}^{\pi}\left|H\left(e^{j \omega}\right)\right|^{2} d \omega
$$

Where $\omega_{\mathrm{s}}$ is the stop band frequency edge.

In the analysis bank, the stopband attenuation cost helps in improving the signal decorrelation and decreasing the amount of aliasing.

$$
C_{\text {analysisstop }}=\sum_{i=0}^{M-1} \int_{\omega \in \Omega_{\text {stopband }}} W_{i}^{a}\left(e^{j \omega}\right)\left|H_{i}\left(e^{j \omega}\right)\right|^{2} d \omega
$$

In the synthesis bank, synthesis filters covering low-frequency bands need to have high stopband attenuation near and/or at to enhance their smoothness. The biased weighting is enforced using two simple linear functions.

$$
C_{\text {synthesisstop }}=\sum_{i=0}^{M-1} \int_{\omega \in \Omega_{\text {stopband }}} W_{i}^{s}\left(e^{j \omega}\right)\left|F_{i}\left(e^{j \omega}\right)\right|^{2} d \omega
$$

Also, the maximum level of aliasing in Pseudo QMF banks can be kept small by keeping Es sufficiently large for $\omega \geq \frac{\pi}{M}$

\subsubsection{Coding Gain}

Coding Gain In image compression designed M-band cosine modulated filter bank used as transform should have high coding gain. As transforms with higher coding gain tend to compact more energy into a fewer number of coefficients. Since in EZW coder progressive transmission is there, higher coding gain almost always translates to higher image quality in the mean-squared sense. Filter Bank's presented in this paper are obtained with a version of the generalized coding gain formula [15]:

$$
E_{\text {codinggain }}=10 \log _{10} \frac{\sigma_{x}^{2}}{\left(\prod_{k=0}^{M-1} \sigma_{x_{i}}^{2}\left\|f_{i}\right\|^{2}\right)^{1 / M}}
$$

$\sigma_{x_{i}}^{2}=h_{i}^{t} R_{x x} h_{i}$. Where $\sigma_{\mathrm{x}}{ }^{2}$ is variance of the input signal; $\sigma_{x_{\mathrm{i}}}{ }^{2}$ is variance of the $\mathrm{i}^{\text {th }}$ subband, $\left\|f_{i}\right\|^{2}$ is norm of the $i^{\text {th }}$ synthesis filter. The signal is the commonly-used AR (1) process with intersample autocorrelation coefficient $\rho=.95$.

\subsubsection{Low DC Leakage measure}

Low DC Leakage measures the amount of dc energy that leaks out to the bandpass and highpass subband. This function concentrates all signal energy at dc into the dc coefficients and thus improves both signal decorrelation and discontinuities in the reconstructed signals. The dc cost function is defined as 


$$
C_{D C}=\sum_{i=1}^{M-1} \sum_{n=0}^{L-1} h_{i}(n)
$$

Notice that all antisymmetric filters have a zero at dc therefore; symmetric filters are applied to reduce the complexity of the optimization process.

Low dc leakage is not as essential to the coder's objective performance as coding gain. However, it improves the visual quality of the reconstructed image significantly.

\section{DESIGN ALGORITHM}

In our design, we search prototype filter coefficients $h$ that minimise the weighted combinatorial cost function. To confine the search process in a feasible solution space, we impose a limit to the perfect reconstruction violation measure. We have to determine only half of the coefficients since the remaining coefficients are obtained, by applying symmetry.

Filter Banks presented in this paper are obtained from the multivariable population based stochastic optimization technique (PSO) developed in 1995, inspired by social behaviour of bird flocking or fish schooling [16]. It utilizes a population of particles called swarms, which fly in the given problem space. In every iteration, each particle is updated by two values: the best solution or fitness that has achieved so far termed $\mathrm{p}_{\text {best }}$. Another value, called $\mathrm{g}_{\text {best }}$ obtained so far by any particle in the population. After finding these two values, the particles adjust its velocity and position. To initialize the algorithm, we set the coefficients to satisfy unit energy constraint. A set of weighting factors that we have found to provide reasonable tradeoffs between various transform properties is $\{10.0,1.0,0.5,0.5$ and 0.1$\}$.

Our problem is formulated as follows:

$$
C_{\text {overall }}=k_{1} C_{\text {codinggain }}+k_{2} C_{\text {analysisstop }}+k_{3} C_{\text {synthesisstop }}+k_{4} C_{D C}
$$

Subject to the constraint:

$$
P R V<\beta
$$

$\beta$ is a small value (e.g. of order $10^{-6}$ ) used here to maintain NPR filter banks for optimizing the objective function by the PSO technique. Compared to the other optimization techniques, PSO has following several advantages [16]:

- It is not affected by the size and nonlinearity of the problems and it converse to solution when other analytical methods fail.

-In PSO, few parameters are required to be adjusted, therefore, it is easy to implement and there are few parameters to adjust.

-As compared to Genetic algorithm, in PSO, information sharing is one way and every evolution only looks for the best solution. Therefore, all the particles tend to converge to the best solution quickly even in the local version in most cases.

\section{RESULTS AND DISCUSSION}

We have designed 8-channel 16 taps (8x16) and 8-channel 24 taps (8x24) pseudo qmf bank optimizing eqn.(9) using PSO technique, to be used as a replacement for the DWT transform at the transforming stage in EZW coder. The results of the filter bank in the form of Magnitude 
response are as shown in Fig.2 (a) and (b). As the filter taps increase stopband attenuation of filter bank is increased from $20 \mathrm{~dB}$ to $32 \mathrm{~dB}$ with slight dip in the lowpass subband filter. This dip results in increased dc leakage as the number of taps increased with increased computational

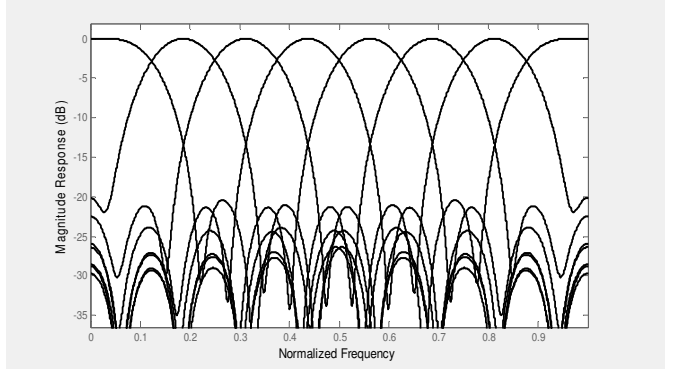

(a)

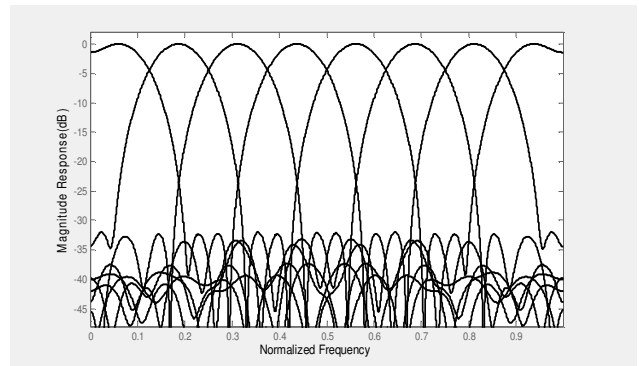

(b)

Figure 2.Frequency response characteristics of (a) 8x16 Pseudo QMF bank (b) 8x24 Pseudo QMF bank

Complexity. However, as the stopband attenuation increased coding gain can not be made high. Inclusion of low dc leakage condition in the objective function with lower $\beta$ constraint visual quality of the image improves but at the cost of reduced PSNR. It can be seen in Table 1 that low dc leakage condition improves the performance of subband filters and reduce the aliasing errors as all the subband filters except the lowpass one, has at least one zero at $\mathrm{z}=1$ and thus all other bandpass and highpass filters are dc transmission free [12]. This constraint is particularly beneficial in EZW coder as the significant coefficients having most of the information are concentrated in the lowest subband and get transmitted first. Thus improve the system efficiency. Performance parameters of the designed $\mathrm{M}$ channel pseudo QMF bank are tabulated in table 1 shows that our filter bank provides higher coding gain as compared to other existing DCT and LOT transform with lower number of taps. Stopband attenuation for the designed filter bank can not be made higher due to highest priority given to coding gain and inclusion of low dc leakage condition in addition to lower $\beta$ (overall distortion) constraint. It can also be seen that the relaxing perfect reconstruction condition increases the feasible solution search space and reduce time of convergence.

Table 1 Comparison of Different Transform Properties

\begin{tabular}{|l|l|l|l|}
\hline \multirow{2}{*}{ Transform Property } & \multicolumn{3}{|c|}{ Transform } \\
\cline { 2 - 4 } & $\begin{array}{c}\text { DCT } \\
(8 \times 8)\end{array}$ & $\begin{array}{c}\text { LOT } \\
(8 \times 16)\end{array}$ & $\begin{array}{l}\text { Proposed filter bank } \\
(8 \times 16)\end{array}$ \\
\hline Coding Gain & 8.83 & 9.22 & 9.96 \\
\hline Stopband Attenuation (dB) & 9.96 & 19.38 & 20.0 \\
\hline DC Attenuation (dB) & 310.62 & 312.5 & 303.32 \\
\hline
\end{tabular}

Coder performance is evaluated on two popular 'Lena' 256x256 and 'Barbara' 512x512 8-bit gray-scale test images. Our designed filter bank used as transform in both standard JPEG in place of DCT and in place of DWT in embedded zerotree coder (EZW). While using EZW 3 level decomposition of the block transform is used as in [8] using wavelet block transform analogy. 
Signal \& Image Processing : An International Journal (SIPIJ) Vol.3, No.4, August 2012

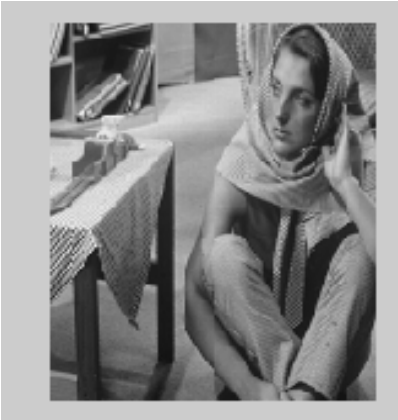

(a)

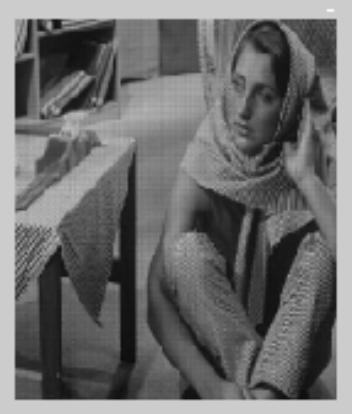

(b)

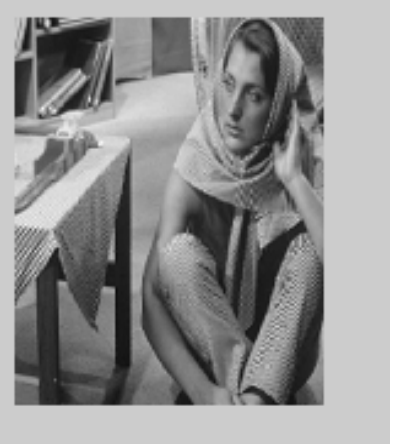

(c)

Figure 3. (a) Original 'Barbara' image (b) reconstructed image with JPEG based coder (c) Reconstructed image with EZW coder at 0.25 bpp.

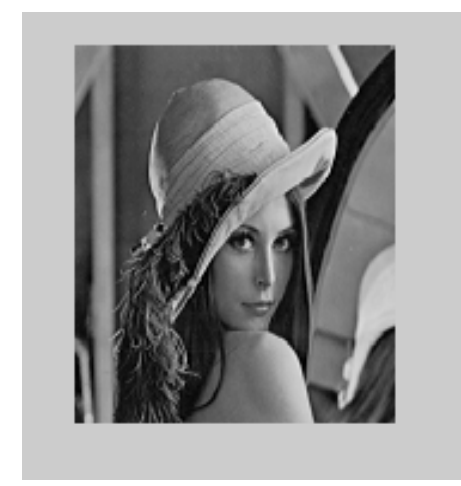

(a)

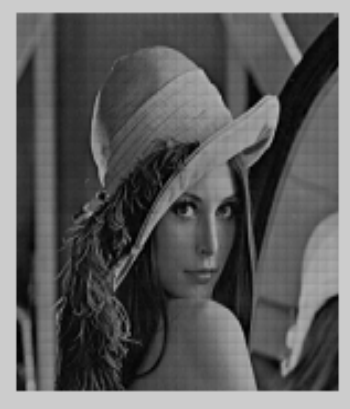

(b)

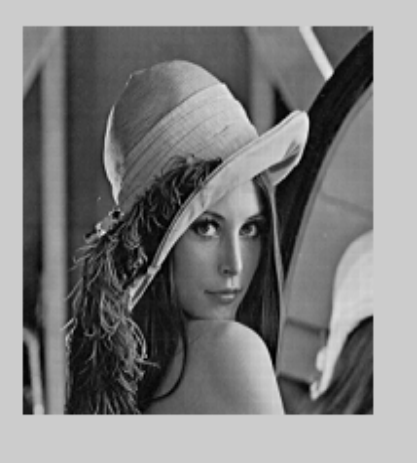

(c)

Figure 4. (a) Original 'Lena' image (b) reconstructed image with JPEG based coder (c) Reconstructed image with EZW coder at 0.25 bpp.

Visual quality of both the images can be easily justify the superiority of the designed filter bank as the blocking artifacts are reduced in JPEG based coder while EZW coder provide better image quality at lower bit rate (0.25bpp). Fig. 3 and Fig. 4 confirm the superiority of the coder using designed filter bank. Comparing to JPEG, blocking artifacts are already remarkably reduced in the DCT-based and the LOT-based coder. Blocking is completely eliminated when the DCT and the LOT are replaced by the new FB's as shown in Figs. 3(b), (c) and 4(b), (c). As the bit rates are further reduced EZW coder consistently performs better. Comparing to the 9/7-tap wavelet, our M-channel filter banks yield overall sharper reconstructed images with more defining edges and more evenly reconstructed texture regions.Comparative results for coder performance in terms of PSNR can be easily judged from Table 2.

The objective distortion measure is the popular peak signal-to-noise ratio (PSNR)

$$
P S W R=10 \log _{10}\left(\frac{255}{M S E}\right) d B
$$

Where, MSE denotes the mean squared error.

As the compression ratio increased PSNR become higher with EZW coder as compared to JPEG (dct based) coder using designed filter bank in place of DWT and DCT respectively. Although the PSNR difference is not as striking, improvement in perceptual quality is rather significant as shown in Figure 3(a)-(c) and Figure 4(a)-(c).As the compression ratio increased PSNR in EZW coder using proposed filter bank educed but still remain higher as compared to other coders. Thus 
Signal \& Image Processing : An International Journal (SIPIJ) Vol.3, No.4, August 2012

the present coder confirms its superiority. However, interblock dependencies are not considered in the coder, but it can be added in the future work.

Table 2 Coding Results (PSNR in $\mathrm{dB}$ ) for Lena

\begin{tabular}{|l|l|l|l|}
\hline Comp. ratio & \multicolumn{3}{|c|}{ Different EZW coder } \\
\hline & DCT & LOT & proposed \\
\hline $1: 8$ & 39.91 & 40.05 & 40.43 \\
\hline $1: 16$ & 36.38 & 36.72 & 37.73 \\
\hline $1: 32$ & 32.90 & 33.56 & 34.27 \\
\hline $1: 64$ & 29.67 & 30.48 & 31.18 \\
\hline
\end{tabular}

\section{CONCLUSIONS}

We have presented a progressive transmission image compression method where M-channel pseudo QMF filter banks and the zerotree entropy coder are combined to get an excellent performance in coding. The designed coder offers higher coding gain and improves visual quality images due to finer frequency partitioning and higher energy compaction. The coder possess advantages of both block transform (region of interest coding /decoding) and progressive image transmission (embedded quantization, exact bit rate control, and idempotency). Finally, the both subjective and objective quality of the system outperforms.

\section{REFERENCES}

[1] V.S. Shingate, T. R. Sontakke \& S.N. Talbar, (2010) "Still Image Compression using Embedded Zerotree Wavelet Encoding”, International Journal of Computer Science \& Communication”, Vol. 1, No. 1, pp.21-24

[2] J. M. Shapiro, (1993) "Embedded image coding using zerotrees of wavelet coefficients," IEEE Trans. Signal Processing, vol. 41, pp. 3445-3462.

[3] Arafael C. Gonzalez, Richared E. Woods, Steven L.Eddins, (2008), Digital Image Proceeing Using MATLAB, Pearson Education

[4] David Salomon, (2004) Data Compression - The Complete Reference, Springer, 3rd edition.

[5] K. Sayood, (2000) Introduction to Data Compression, 2nd Ed, Academic Press, Morgan Kaufmann publishers

[6] R. A. DeVore, B. Jawerth, and B. J. Lucier, (1992) "Image compression through wavelet transform coding," IEEE Trans. Inform. Theory, vol. 38, pp.719-746.

[7] H.S.Malvar,( 1998) "Biorthogonal and nonuniform lapped transforms for transform coding with reduced blocking and ringing artifacts," IEEE Trans. Signal Processing, Spec. Issue Multirate Syst., Filter Banks, Wavelets, Applications. vol. 46, pp. 1043-1053.

[8] Z. Xiong, O. Guleryuz, and M. T. Orchard, (1996) “A DCT-based embedded image coder,” IEEE Signal Processing Lett., vol. 3, pp. 289-290.

[9] Janaki. R, Dr.Tamilarasi (2011) "Still Image Compression by Combining EZW Encoding with Huffman Encoder", International Journal of Computer Applications (0975 - 8887), Volume 13, No.7, pp.1-7. 
Signal \& Image Processing : An International Journal (SIPIJ) Vol.3, No.4, August 2012

[10] J. Kennedy and R. Eberhart, (1995) "Particle swarm optimization," in proceeding of IEEE international conference on neural networks (ICNN), vol. 4, pp. 1942-1948.

[11] P. P. Vaidyanathan, (1993) Multirate Systems and Filter Banks. Englewood Cliffs, NJ: Prentice- Hall.

[12] T. D. Tran and T. Q. Nguyen, (1997) "On M-channel linear-phase FIR filter banks and application in image compression,” IEEE Trans. Signal Processing, vol. 45, pp. 2175-2187.

[13] M. Rossi, J.Yun Zhan, W.Steenaart, (1996) "Iterative constrained least squares design of near perfect reconstruction pseudo qmf banks", CCECE, IEEE, pp.766-769.

[14] A. Boukhobza, A. Bounoua, A. Taleb Ahmed, N. Taleb, (2009) "A Filter Banks Design Using A Multiobjecive Genetic Algorithm For An Image Coding Scheme”, ICIP IEEE ,pp.1933-1936.

[15] J. Katto and Y. Yasuda, "Performance evaluation of subband coding and optimization of its filter coefficients,” SPIE Proc. Vis. Common. Image Process. 1991.

[16] A. Kumar, G. K. Singh, and R. S. Anand, (2009) "Design of Quadrature Mirror Filter Bank using Particle Swarm Optimization (PSO)",International Journal of Recent Trends in Engineering, Vol 1, No. 3, pp.213-217.

[17] J. Kennedy and R. Eberhart, (1995) "Particle swarm optimization," in proceeding of IEEE international conference on neural networks (ICNN), vol. 4, pp. 1942-1948.

\section{Authors}

Dr. Aditya Goel B.E. ( Hons), M.Tech (I.I.T. Bombay) and PhD in 2000. Teaching \& Research experience of 22 years. Presently working as Professor \& H.O.D., Electronics \& Communication at National Institute of Technology, (MANIT) Bhopal. He has publish-ed more than 60 Research papers in reputed International Journals \& Conferences inclu-ding IEEE. His areas of interests are Optical Communication, Digital Signal Processing, and Computer Communication. He has guided several M.Tech theses on these areas. He had been Principal Investigator of the research project titled "Broadband Lightwave com-munication systems" sanctioned by Govt. of India, New Delhi. He worked as Principal Investigator of the R\&D Project "High speed optical components for WDM systems" sanctioned by M.H.R.D. Govt. of India, New Delhi. He has reviewed many research papers of International conferences \& journals including SPIE. He is a Life Fellow member of the Institution of Electronics \& Telecommunication Engineers, Fellow of Institution of Engineers (I.E.), India and Life Senior member of Computer Society of India.

Anamika Jain obtained her B.E degree from Samrat Ashok Technological Institute (SATI) Vidisha (M.P.) in 1992 and M.Tech. in Micro and mm wave from M.A.N.I.T Bhopal M.P. in 2003. She is pursuing her PhD. in Deptt. of Electronics and Telecommunication Engineering of MANIT Bhopal (M.P.). She is working as a senior lecturer in Electronics and Telecommunication Engineering department of S.V.Polytechnic Bhopal..

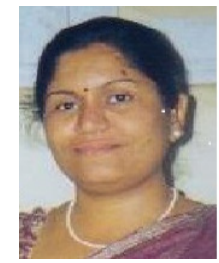

